\title{
MORBILIDAD MATERNA EXTREMA AGUDA POR PREECLAMPSIA SEVERA
}

\section{EXTREME MATERNAL MORBIDITY IN SEVERE PREECLAMPSIA}

\author{
C.E.R ${ }^{1}$ Carlos Mata Hernández ${ }^{2}$, Nolvia Aguilar ${ }^{3}$
}

1 Comité Editorial de la Revista de Postgrados de Medicina UNAH, Dirección de Docencia e Investigación-HE: Dr. Carlos Vargas Pineda, Dr. Rolando-Aguilera-L, Dra. Guadalupe-Romero-A

2Médico Residente III, Universidad Nacional Autónoma de Honduras, Postgrado de Ginecología y Obstetricia.

3Médico Ginecoobstetra, Jefe del Servicio de Emergencia de Ginecoobstetricia del Hospital Materno-Infantil

\section{Resumen}

La Preeclampsia severa y sus complicaciones están asociadas a un aumento de la morbilidad, mortalidad materna y perinatal. Objetivo: Se identificó si los casos de Morbilidad Materna Aguda Extrema en Preeclampsia Severa eran prevenibles o no, en el Bloque Materno Infantil del Hospital Escuela, desde el 01 de Enero del 2009 al 31 de Enero del 2010. Métodos: Fué un estudio descriptivo transversal, se tomó una muestra por conveniencia de 128 pacientes que cumplieron con la definición de preeclampsia Severa, se analizó la presencia o no de morbilidad materna extrema, las diferencias entre cada grupo, la presencia de las complicaciones graves y condiciones asociadas a a la presencia de estas complicaciones en las pacientes con preeclampsia severa. Resultados: De las 128 casos que cumplieron con el criterio de inclusión, solo 13 casos presentaron morbilidad materna extrema (10.2\%). La complicación más frecuentes fué el Síndrome de Hellp (84.6\%). El mayor número de casos se presentó en el grupo de pacientes con edades menores de 20 años (46.2\%) y en las primigestas (61.5\%), con una mortalidad del recién nacido de un $38.5 \%$. La falla en la prevenibilidad más frecuente fué falla en el seguimiento al egreso de la paciente (proveedor) en un 100\%.Conclusión: Se estableció que los casos de preeclampsia severa que presentaron complicaciones eran prevenibles.

Palabras claves: preeclampsia severa, morbilidad materna extrema, complicaciones, prevenibilidad.

Abstract: Severe preeclampsia and its complications are associated with increased morbidity, maternal and perinatal mortality.Objective: To identify whether cases of severe acute maternal morbidity in severe preeclampsia were preventable or not, in Block School 
Mother and Child Hospital, from January 1, 2009 to January 31, 2010. Methods: It was a descriptive study, it was a convenience sample of 128 patients who met the definition of severe preeclampsia, we analyzed the presence of extreme maternal morbidity, the differences between each group, the presence of serious complications conditions attached to a the presence of these complications in patients with severe preeclampsia.

Results: Of the 128 cases that met the inclusion criteria, only 13 cases had extreme maternal morbidity (10.2\%). The most frequent complication was the HELLP syndrome (84.6\%). The largest number of cases were in the group of patients aged under $20(46.2 \%)$ and in primigravida (61.5\%), with a mortality rate of newborn $38.5 \%$. The failure was most common preventability failure to follow the discharge of the patient (provider) by $100 \%$. Conclusion: It was established that cases of severe preeclampsia complications were preventable.

Keywords: severe preeclampsia, extreme morbidity, complications, preventability.

\section{Introducción}

La Preeclampsia severa y sus complicaciones están asociadas a un aumento de la morbilidad, mortalidad materna y perinatal. $^{1}$ En países desarrollados las complicaciones de la Preeclampsia se asocian a riesgo de muerte materna de 0 a $1.8 \%{ }^{2,3}$ Por otro lado en los países en vías de desarrollo se ha descrito una tasa de mortalidad alta de hasta $14 \%{ }^{3,13}$ La alta mortalidad reportada en estos países, ha sido notada primariamente en pacientes sin control prenatal o con eventos como los de eclampsia, a distancias considerables fuera de las áreas hospitalarias. ${ }^{13}$ En adición estas altas tasa tanto de morbilidad como mortalidad puede atribuirse a escasos recursos hospitalarios y carencia de facilidades de atención en una unidad de cuidados intensivos para el manejo de la Preeclampsia severa y sus complicaciones. ${ }^{4,5}$ En un estudio, otros autores encontraron que el riesgo de muerte aumentaba con la edad (mujeres de 30 años o más), mujeres sin control prenatal, mujeres de raza negra, o embarazos menores de 28 semanas. ${ }^{6} \quad$ La morbi mortalidad perinatal se mantiene alta en pacientes con preeclampsia severa, reportándose muerte perinatal del $5.6 \%$ a $11.8 \%$ esta alta tasa de muerte perinatal está relacionada a prematuridad, abruptio placentae, y severa restricción del crecimiento intrautero. ${ }^{7,12}$ En un estudio multicéntrico, sobre morbilidad materna extrema (near-miss), realizado en 26 hospitales de Centroamérica, se demostró que la principal causa de morbilidad severa en la mujer gestante en Centroamérica, son los trastornos hipertensivos. Un $45 \%$ de los casos de near-miss (213/472),

fueron secundarios a trastornos hipertensivos (Preeclampsia 19.7\%, Eclampsia 19.1\% y síndrome de HELLP 5.1\%). Además, un $71.8 \%$ de las causas de la morbilidad 
extrema eran prevenibles. $^{15} \quad$ La presente investigación pretende registrar los casos de Preeclampsia Severa, sus complicaciones y relación con la mortalidad materna con el objetivo de identificar si los casos de Morbilidad Materna Aguda Extrema en Preeclampsia Severa son prevenibles o no, en el Hospital Escuela, Bloque Materno Infantil desde el 01 Enero 2009 a 31 Enero 2010.

\section{Materiales y métodos}

Fue un estudio descriptivo transversal, se revisaron los expedientes de la Sala de Labor y Parto del Bloque Materno Infantil del Hospital Escuela desde el 01 Enero 2009 a 31 Enero 2010 obteniendo la muestra por conveniencia de 128 pacientes que ingresaron con embarazo mayor de 20 semanas con:

- Presión arterial sistólica mayor o igual que $160 \mathrm{mmHg}$

- Presión arterial diastólica mayor o igual que $110 \mathrm{mmHg}$

- Proteinuria mayor de 5 gr. en 24 horas o $3(+)$ por tira reactiva

- Cefalea intensa persistente

- Cambios visuales persistentes

- Tinnitus persistente

- Hiperreflexia/clonus

- Dolor epigastrio y/o en cuadrante superior derecho

- Trombocitopenia

- Elevación de enzimas hepáticas

- Síndrome de HELLP

- Edema agudo de pulmón

- Oliguria/Insuficiencia renal aguda

- Eclampsia/Complicación neurológica

- Restricción del crecimiento fetal/Muerte fetal
- Hematoma de hígado Se ingresó al estudio, toda paciente con criterios clínicos y/o de laboratorios de preeclampsia severa, con 0 sin complicaciones, admitida en el Servicio de Obstetricia del Hospital participante. Se excluyeron del estudio las pacientes con Enfermedad del Trofoblasto o con patología médica asociada. Las variables estudiadas fueron: Preeclampsia severa (dependiente), edad materna, paridad, edad gestacional de inicio de control prenatal, control prenatal, procedencia de la paciente, edad gestacional a la que se diagnostica la preeclampsia, edad gestacional al momento de la interrupción, Test de Apgar, peso del recién nacido, condición de egreso del recién nacido (vivo vs muerto), tratamiento aplicado, preeclampsia severa complicada (morbilidad materna aguda extrema o Near-Miss),condición de egreso de la madre (viva o muerta). Se diseñó un instrumento para la recolección de la información, el cual fué validado en el $10 \%$ de las pacientes que no se incorporaron al estudio y los resultados obtenidos del estudio se manejaron en una base de datos utilizando Epi Info 3.5.1, los cuales se expresaron en frecuencias, porcentajes y medidas de tendencia central.

\section{Resultados}

De las 128 pacientes que cumplieron con el criterio de inclusión de preeclampsia severa, solo 13 presentaron morbilidad materna extrema (10.2\%). Ver tabla \# 1 
TABLA \# 1

PRESENCIA DE MORBILIDAD MATERNA EXTREMA

\begin{tabular}{|c|c|c|}
\hline $\begin{array}{c}\text { Morbilidad Materna } \\
\text { Extrema Aguda }\end{array}$ & Frecuencia & Porcentaje \\
\hline Si & 13 & $10.2 \%$ \\
\hline No & 115 & $89.8 \%$ \\
\hline Total & 128 & $100.0 \%$ \\
\hline
\end{tabular}

El mayor número de casos se presentó en el grupo de pacientes con edades menores de 20 años (46.2\%) siendo más frecuente en el grupo de las primigestas (61.5\%), procedentes del área urbana (69.2\%), con una edad gestacional antes de las 36 semanas de gestación (61.5\%), obteniendo recién nacidos con peso menor de 1500 gramos (53.8\%) y una mortalidad del recién nacido de un $38.5 \%$ en pacientes con morbilidad materna extrema.

TABLA \# 2

CONDICION DEL RECIEN NACIDO AL EGRESO Y PRESENCIA DE MORBILIDAD MATERNA EXTREMA

MORBILIDAD MATERNA EXTREMA

\begin{tabular}{|c|c|c|c|}
\hline CONDICION AL EGRESO & Si & No & TOTAL \\
\hline MUERTO & $38.5 \%$ & $61.5 \%$ & 100 \\
\hline VIVO & $7 \%$ & $93 \%$ & 100 \\
\hline
\end{tabular}

La complicación más frecuentes fue el Síndrome de Hellp (84.6\%) Ver tabla \# 3

TABLA \# 3

PRINCIPALES COMPLICACIONES GRAVES

\begin{tabular}{|c|c|c|}
\hline $\begin{array}{c}\text { COMPLICACIONES } \\
\text { GRAVES }\end{array}$ & Frecuencia & Porcentaje \\
\hline Síndrome de Hellp & 11 & $84.6 \%$ \\
\hline Eclampsia & 1 & $7.7 \%$ \\
\hline $\begin{array}{c}\text { Desprendimiento } \\
\text { prematuro de placenta }\end{array}$ & 1 & $7.7 \%$ \\
\hline \begin{tabular}{c|c|c|}
\hline Total \\
\hline
\end{tabular} & 13 & $100.0 \%$ \\
\hline
\end{tabular}

Se estableció que los casos que presentaron complicaciones eran prevenibles en un $10.2 \%$, siendo las fallas en la prevenibilidad más frecuentes el seguimiento al egreso de la paciente (proveedor) en un $100 \%$ y falla para tratar (proveedor) en un $40 \%$.

\section{Discusión}

La mortalidad materna ha sido el evento de estudio principal para la evaluación y monitoreo de las unidades prestadoras de servicios de salud. En países en vías de desarrollo, a pesar que la mortalidad materna no ha alcanzado cifras bajas, los casos de morbilidad materna extrema ocurren de manera más frecuente que la muerte materna. Otros estudios han logrado determinar que la morbilidad materna extrema aguda es prevenible hasta en un $71.8 \%$, pero nuestro estudio solo se encontró que los casos eran prevenibles en un $10.2 \%$, la diferencia podría deberse a que no se 
tuvo acceso a la cantidad total de expedientes reportados durante el periodo de estudio. Al igual que otros estudios revisados la edad, la paridad, la procedencia, edad gestacional de presentación de la patología, constituyen factores de riesgo para el desarrollo de complicaciones pero con una mayor mortalidad perinatal hasta un $38.5 \%$ debido a la prematurez del embarazo en que se presentaron los casos y que a pesar de brindarles manejo conservador para el cumplimiento de inductores de madurez pulmonar no fue posible la reducción de la misma. Las fallas en la prevenibilidad de complicaciones que llevaron a nuestras pacientes a una morbilidad materna extrema fueron falla en el egreso de nuestras pacientes y falla al momento del tratamiento, por lo cual estos resultados nos permitieron dar las siguientes recomendaciones: dar el seguimiento respectivo en la Consulta Externa de Ginecología de los diferentes centros regionales, centro de salud con medico, clínicas periféricas, a cada una de las pacientes que egresa para saber la evolución clínica de la misma y Brindar jornadas de actualización sobre los trastornos hipertensivos en el embarazo al personal médico y de enfermería para la identificación oportuna de los factores de riesgo que puedan llevar a una paciente a presentar una morbilidad materna extrema.

\section{Referencias}

1. Ronsmans C, Filipi V. Reviewing severe maternal morbidity: learning from women who survive lifethreatening complications. En: Lewis $G$ et al. (eds.) Beyond the Numbers: Reviewing Maternal Deaths and
Complications to Make Pregnancy Safer. Geneva: World Health Organization 2004: 103-124.

2. Stones W, Lim W, Farook A, Kelly M. An investigation of maternal morbidity with the identification of life-threatening "near miss" episodes. Health Trends 1991; 23:13-15.

3. Geller SE, Rosenberg D, Cox SM, et al. The continuum of maternal morbidity and mortality: Factors associated with severity. Am J Obstet Gynecol 2004; 191: 939-44.

4. Geller SE, Rosenberg D, Cox SM, Kilpatrick S. Defining a conceptual framework for near-miss maternal morbidity J Am Med Womens Assoc. 2002 Summer;57(3):135-9.

5. Geller SE, Cox SM, Kilpatrick SJ. A descriptive model of preventability in maternal morbidity and mortality: J Perinatol. 2006 Feb;26(2):79-84.

6. Berg CJ, Harper MA, Atkinson SM, et al. Preventability of pregnancyrelated deaths: Results of a statewide review. Obstet Gynecol 2005; 106: 1228-1234.

7. Geller SE, Rosenberg D, Cox S, Brown M, Simonson L, Kilpatrick S.A scoring system identified near-miss maternal morbidity during pregnancy J Clin Epidemiol. 2005 Sep;58(9):962; author reply 962-3.

8. Reviewing Maternal Deaths and Complications to Make

9. Pregnancy and Childbirth Saber, Making Pregnancy Safer, WHO/HQ Regional Health Forum - Volume 9, Number 1, 2005 
10. JHPIEGO/Programa de Salud Materna y Neonatal y PATH, Prevención de la Hemorragia Postparto: Manejo del Tercer Período del Parto. Número Especial. Outlook. 2002; 19(3)108

11. WHO/Europe web site at http://www.euro.who.int/pubrequest.

12. Ronsmans, C., Filippi, V. Reviewing severe maternal morbidity: learning from survivors of life-threatening complications. Beyond the Numbers: Reviewing maternal deaths and complications to make pregnancy safer complications.OMS 2.004

13. De Sousa JPD, Duarte G, BasileFilho A: Near-miss maternal mortality in developing countries. Eur J Obstet Gynecol Reprod Biol 2002, 104:80.

14. Rodríguez-Iglesias G, Calzado JD, Ribeiro LP: Experiencia de 12 años de trabajo la atención de adolescentes obstétricas críticamente enferma en la unidad de cuidados intensivos. Rev Cubana Obstet Ginecol 1999, 25:141-14

15. Jarquín $G$ et al, morbilidad materna extrema: las casi muertas en la región centroamericana julio a diciembre de 2007, Revista Centroamericana de Ginecología y Obstetricia, Abril 2008, pag: 1-48

16. Mejia Elias. Metodología de Investigación Científica. Cenit editores. Lima, Peru. 2005. pp. 1318

17. SARAVIA, Marcelo. Metodología de Investigación. Universitat de Barcelona. 2001-2004. pp. 1-18. 